\title{
Klimaanlagen, luftführende Systeme, Wartung
}

\author{
Andreas Schmucker, Andreas Kuhnle, Volker Williger, Reinhard Hoffmann
}

\section{Zusammenfassung}

Neueste Erkenntnisse über die Rolle der Raumluft bei der Infektionsprophylaxe führen zum Überdenken bisheriger OP-Konzepte in bezug auf Raumeinteilung, Organisation und nicht zuletzt der Raumlufttechnik. Bisherige Forderungen nach hohen Luftmengen, Reinraumfilterung aller zur OP-Abteilung gehörenden Räume und einer vergleichsweise hohen Anzahl an den einzelnen OP-Räumen zugeordneten Einund Ausleiträumen können nach diesen Erkenntnissen reduziert werden. Die hygienischen Bedingungen werden hierdurch nicht vermindert. Ein hygienischer, wirtschaftlicher und ener- gieeffizienter Betrieb steht hierbei im mischen Schutzkonzeptes und der sich hieraus ergebenden OP-Struktur ist die sog. Zuluftdecke mit turbulenzarmer Verdrängungsströmung (TAV) im OP-Saal. Die Abmessungen betragen mindestens $2,8 \times 2,8 \mathrm{~m}$. Es wird mit relativ großen in die Nachbarräume überströmenden Luftmengen gearbeitet, so dass auf Luftschleusen verzichtet werden kann und alle hygienisch relevanten Handlungen im Schutzbereich vorgenommen werden können. Bei dem Neu- und Umbauprojekt der Berufsgenossenschaftlichen Unfallklinik in Frankfurt werden diese Konzepte umgesetzt. Vordergrund. Grundlage dieses dyna-

\section{Raumkonzeption}

Die neue OP-Abteilung im 1.Obergeschoss der Berufsgenossenschaftlichen Unfallklinik in Frankfurt wird nach den neuesten Erkenntnissen der Anästhesiologie und der Krankenhaus- Hygiene geplant und entwickelt (Abb.5).

Einleit-, Ausleit- und Waschräume werden nicht den einzelnen OP-Sälen zugeordnet. Für je 3 OP-Säle stehen 2 Einleitungsräume zur Verfügung.

Alle OP-Säle weisen den gleichen Standard auf und gewährleisten so interdisziplinäre Nutzungen sowie den Einsatz moderner EDV-Gestützter Behandlungsverfahren.

Die Sterilgutversorgung und OP-Vorbereitung wurde auf der Nord- und Westseite des neuen OP-Zentrums angeordnet. Ein Aufzug stellt die direkte Verbindung zur Zentralsterilisation im Untergeschoss her. aufwändige Raumkonzepte und raumlufttechnische Anlagen. Die Entwicklung von effizienteren Lüftungsdecken, die den hygienisch relevanten Schutzbereich abschirmen (Abb.1 -4) führt zur Verkleinerung der bisherigen aufwändig herzustellenden OP-Bereiche.
Eine große Holding („Prä- und Postoperative Raumzone") für die stationären und die ambulanten Operationen wurde in unmittelbarer Nähe vorgesehen. Eine
Ziel aller Maßnahmen bei Eingriffen ist die Minimierung des Risikos postoperativer Infektionen. Die Luftqualität im OPFeld spielt allerdings eine untergeordnete Rolle, vielmehr ist der Raumluftströmung bzw. -richtung eine entsprechende Rolle zu zuweisen. V.a. sind Kontaminationen durch aufgewirbelte Schwebstoffe, z.B. vom Boden zum Eingriffsbereich und zum OP-Besteck zu vermeiden. Kontaminationen über die Luft aus OP-Nebenbereichen sind eher unwahrscheinlich.

Aufgrund der bisher angewandten OPLüftungssysteme im hygienischen

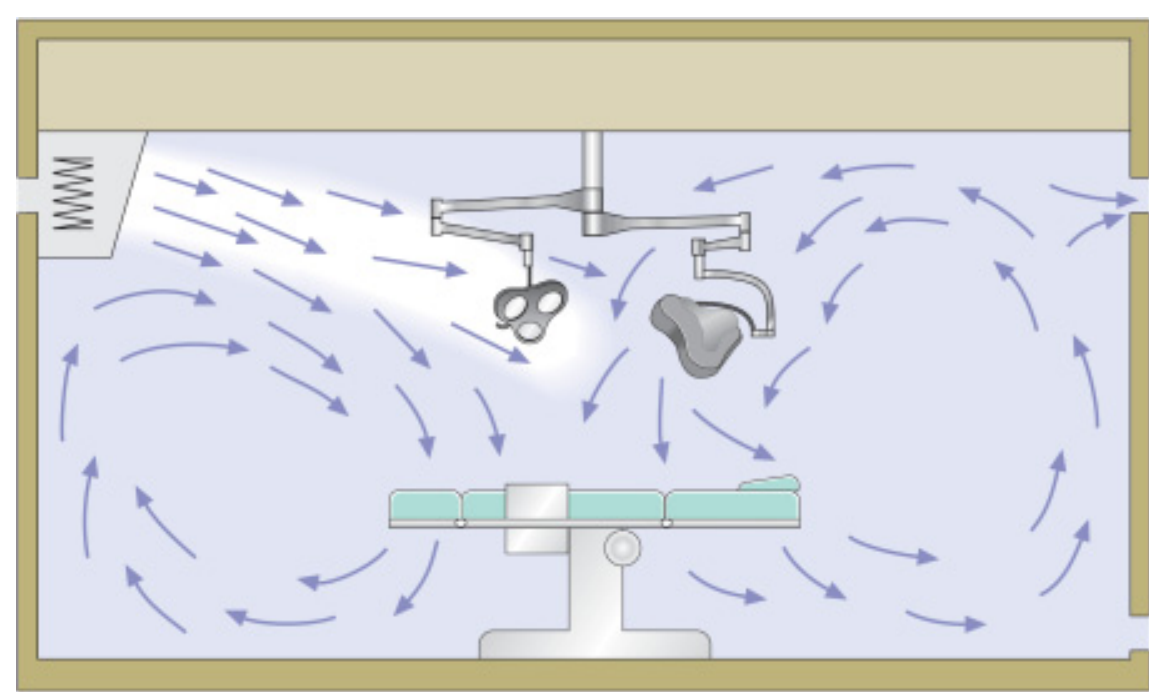

Abb. 1 Hygieneauslass 


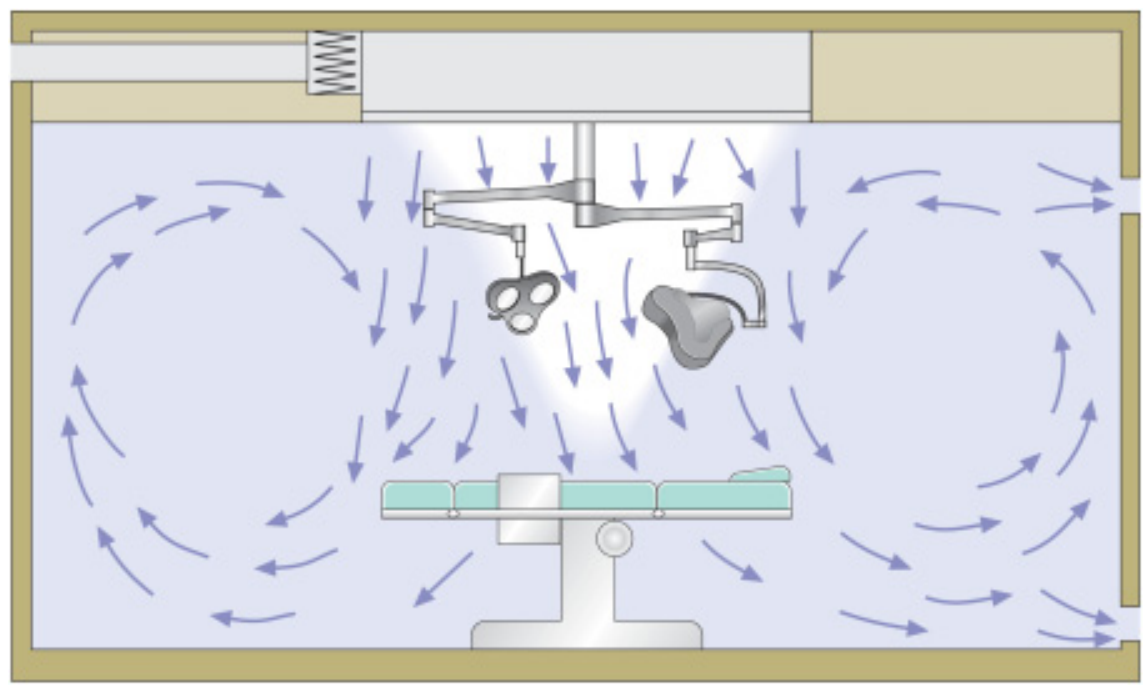

Abb. 2 OPL-Decke

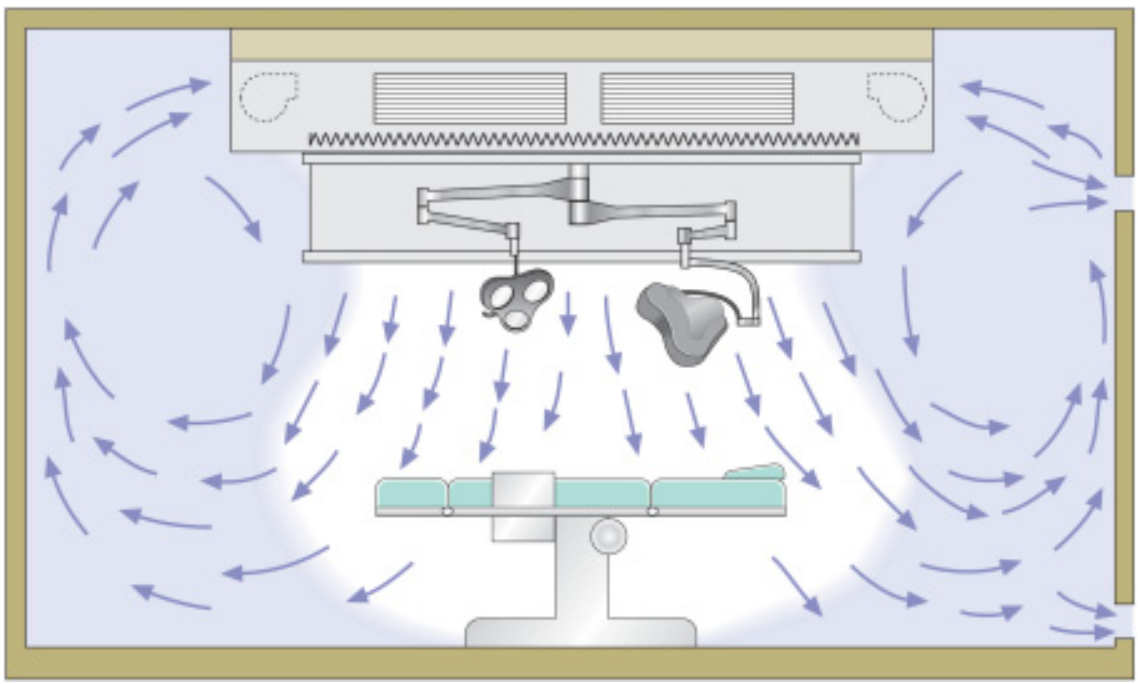

Abb. 3 Stützstrahldecke

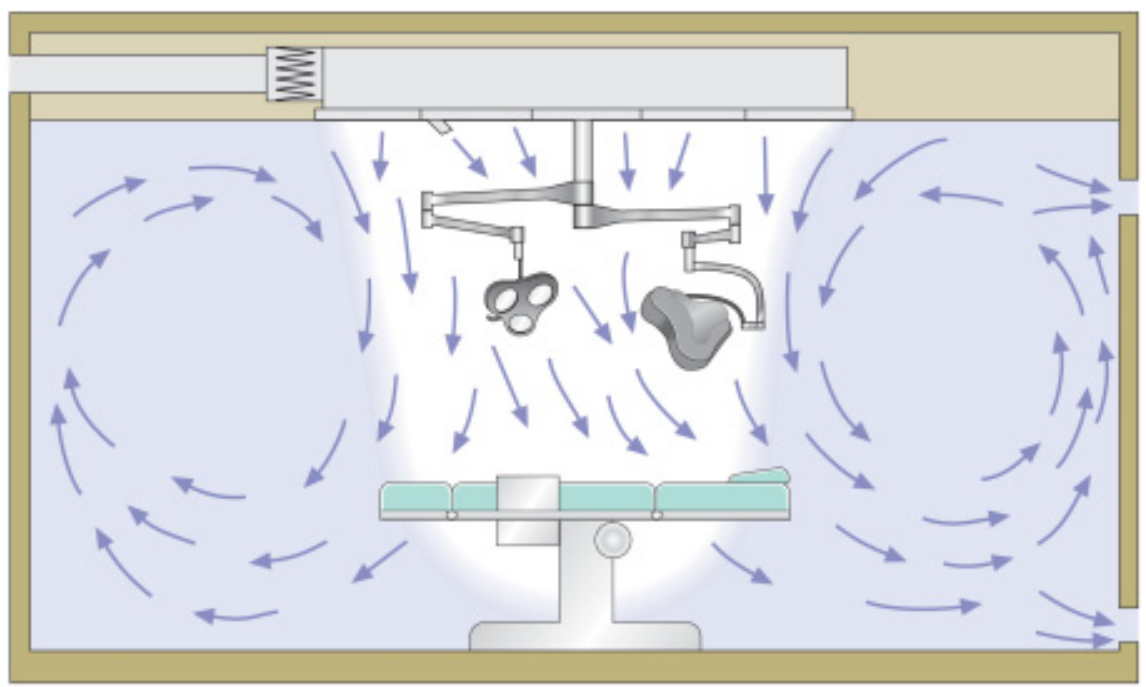

Abb. 4 Verdrängungsdecke wechselseitige Nutzung für den OP-Betrieb ist möglich.

\section{Luftführungssystem}

Durch raumlufttechnische Einrichtungen von OP-Abteilungen in Kliniken werden folgende Ziele verfolgt:

- Hygienetechnische Schutzfunktion gegenüber aerogen übertragenen Infektionserregern

- Erfüllung der Anforderungen der Arbeitshygiene (z.B. Einhaltung MAKWerte/Behaglichkeit)

- Effektive Nutzung der Flächen/Kubatur

- Minderung der Betriebskosten unter Beachtung der Investitionskosten

- Minimierung der Einschränkungen durch raumlufttechnische Einrichtungen.

Diese Ziele werden bei der neuen OP-Abteilung in der BG Unfallklinik Frankfurt wie im Folgenden dargestellt realisiert.

Um die hygienetechnische Schutzfunktion der raumlufttechnischen Einrichtungen gegenüber durch die Luft übertragener Infektionserreger während der Operation zu verbessern, werden OP-Decken mit turbulenzarmer Verdrängungsströmung (TAV) mit einer Größe von 3,2 $\times 3,2 \mathrm{~m}$ vorgesehen. Der Vorteil dieses Prinzips gegenüber der herkömmlichen turbulenten OP-Lüftungstechnik besteht darin, dass die Infektionserreger in geringerem Umfang im gesamten OP-Raum verteilt werden. Weiterhin wird der Eintrag von Infektionserregern auch aus angrenzenden Bereichen minimiert. Durch die leistungsfähigeren OP-Decken mit turbulenzarmer Verdrängungsströmung und Gewebe-Laminisatoren (LaminarAir-Flow) und einer entsprechenden Größe wird gewährleistet, dass sich unter anderem auch die Instrumententische im sterilen Bereich befinden. Ferner kann auf die bisher für die Schutzdruckhaltung erforderlichen Luftschleusen, Sterilflure sowie separaten Ein- und Ausleiträume verzichtet werden, bzw. sind diese nicht mehr einem definierten OP zugeordnet, sondern dienen somit der Patientenvorbereitung für mehrere OP's.

Durch die Vergrößerung des OP-Deckenfeldes wird nunmehr ein ausreichend großer OP-Schutzbereich erzielt, um die über die Raumluft übertragenen Keime von der Operationswunde des Patienten und dem Instrumententisch fernzuhalten (Abb. 6).

Ein weiteres Kriterium für die planerische Entscheidung im Hinblick auf die 


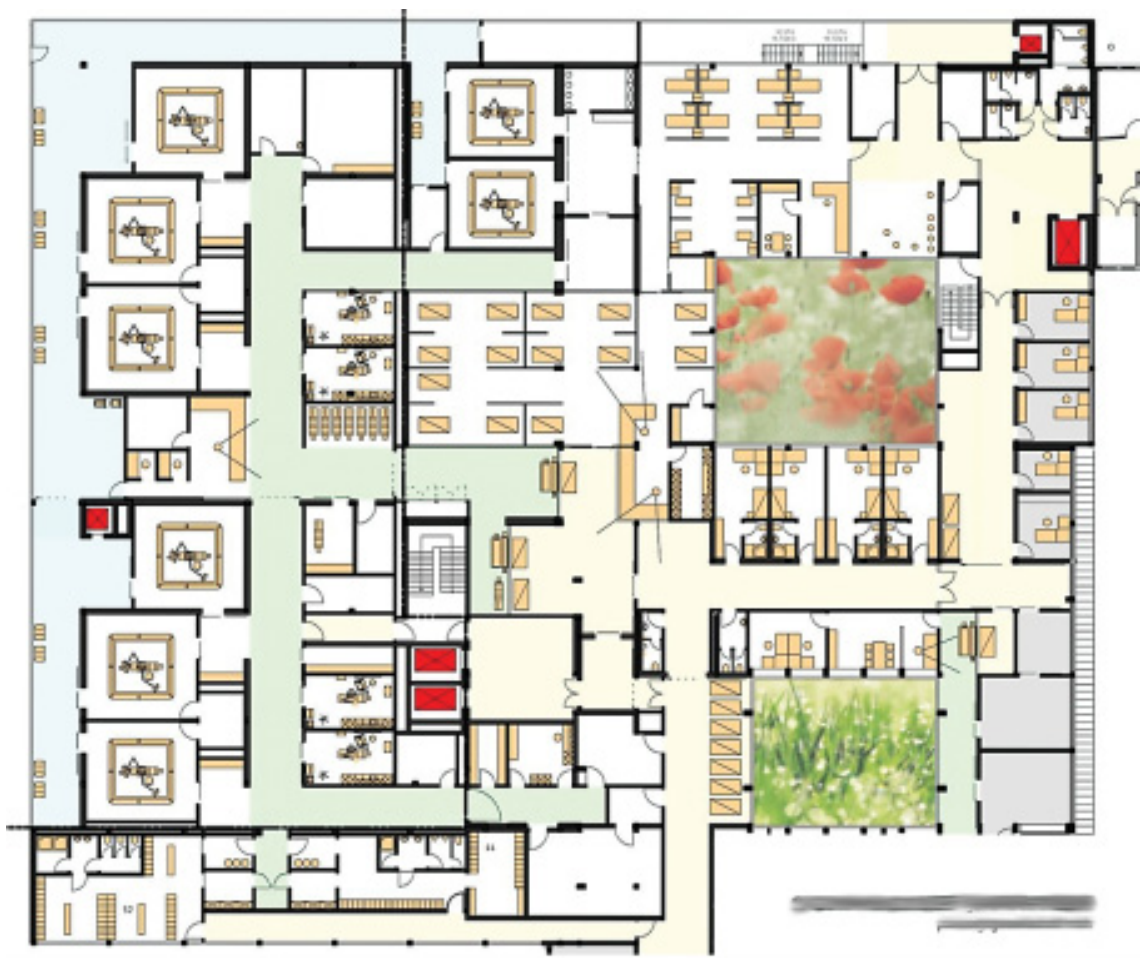

Abb.5 Neue OP-Abteilung im 1. OG der BGU.

Größe der OP-Decke von 3,2 × 3,2 m besteht darin, dass die Behaglichkeit für das OP-Team gegenüber kleinflächigeren OP-Decken wesentlich verbessert werden kann.

Um die vollständige Kühllast des OPRaums abzuführen, kommt es beim Einsatz einer kleinflächigen OP-Decke unter Berücksichtigung einer Zulufttemperatur von ca. $18^{\circ} \mathrm{C}$ zu einer Temperaturdifferenz zwischen Zuluft und Abluft von teilweise mehr als 8 Kelvin. Das heißt, das
OP-Team ist während der Operation aufgrund der Temperaturdifferenz Zug-

Im Gegensatz dazu ergibt sich in Verbindung mit der erforderlichen Abströmgeschwindigkeit der OP-Zuluftdecke von $0,25 \mathrm{~m} / \mathrm{s}$ und der verwendeten Größe des OP-Deckenfelds von 3,2 $\times 3,2 \mathrm{~m}$ ein Zuluftvolumenstrom über die OP-Decke von ca. $8000 \mathrm{~m}^{3} / \mathrm{h}$, der sich aus dem gemäß DIN 1946, Teil 4 erforderlichen Außenluftvolumenstrom in Höhe von erscheinungen ausgesetzt.

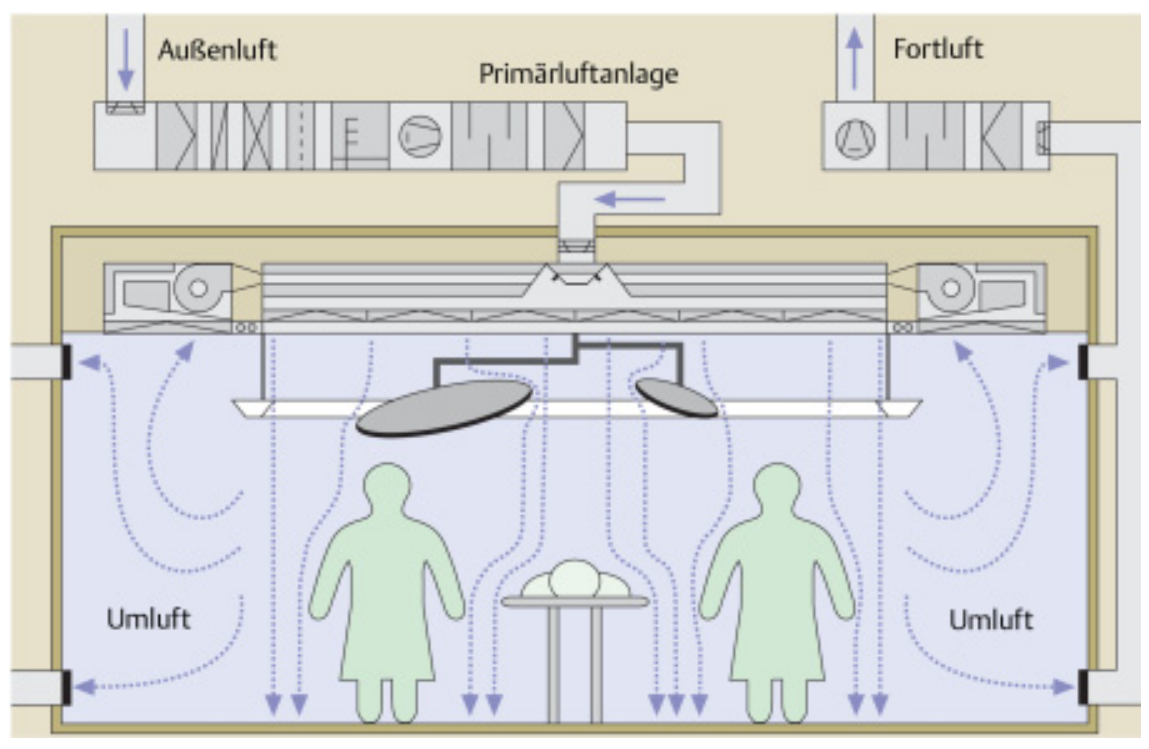

Abb. 6 Prinzipbild
$1200 \mathrm{~m}^{3} / \mathrm{h}$ und dem entsprechenden Umluftanteil zusammensetzt.

Durch die höhere Luftmenge der größeren OP-Decke wird die Temperaturdifferenz zwischen Zuluft und Abluft im OP auf ca. 2 Kelvin verringert und infolgedessen die Behaglichkeit wesentlich verbessert.

Somit werden etwa $75 \%$ der benötigten Luft als Umluft dem Luftverteilerkasten zugeführt, in welchem Umluft und AuBenluft aus der raumlufttechnischen Anlage gemischt werden. Die Umluft wird über einen sogenannten umlaufenden Umluftring angesaugt. Der Geräuschpegel liegt hierbei unterhalb von $35 \mathrm{~dB}(\mathrm{~A})$. Durch die Verdrängungsströmung wird neben der geringen Keimkonzentration von weniger als $10 \mathrm{KBE} / \mathrm{m}^{3}$ zusätzlich ein angenehmes Raumklima für das Personal geschaffen.

Mit Veröffentlichung des Entwurfs der neuen Richtlinie VDI 2167, Teil 1, Technische Gebäudeausrüstung von Krankenhäusern, kann der o.g. Außenluftstrom auf $800 \mathrm{~m}^{3} / \mathrm{h}$ reduziert werden, der Umluftanteil steigt entsprechend. Somit kann eine weitere Investitions- und Energiekosteneinsparung erzielt werden, ohne Beeinträchtigung der hygienischen Anforderungen und der Behaglichkeit. Der Ansatz der o. g. Richtlinie als Grundlage der Planung wird mit den Planungsbeteiligten wie Bauherr, Nutzer, Hygieniker, Behörden, und Architekt abgestimmt.

Durch den Einsatz von großen LaminarAir-Flow-Deckenfeldern kann, wie bereits erwähnt, unter anderem auf den jedem einzelnen OP zugeordneten Ein-/ Ausleitraum, Waschraum und einem vorgeordneten Sterilflur in Raumklasse IAusführung verzichtet werden.

Dies hat zur Folge, dass im Vergleich mit einem klassischen OP-Konzept eine wesentlich geringere Raumgrundfläche von der OP-Abteilung beansprucht wird.

\section{Zentralenanordnung}

Die Aufbereitung der erforderlichen Außenluft für den OP-Bereich erfolgt über eine Raumlufttechnikzentrale im 2.OG, direkt über den OP‘s. Die Ansaugung der Außenluft erfolgt über die Innenhoffassade mit einer entsprechenden Ansaugkammer, in der auch die 1. Filterstufe untergebracht ist.

Die 2. Filterstufe befindet sich in den zwei RLT-Geräten der OP-Abteilung, die je 50\% 
des Zuluftvolumenstroms bereitstellen (Redundanz). Die 3. Filterstufe befindet sich, wie bereits erwähnt, in den jeweiligen OP-Decken. Die Luftverteilung in die OP-Abteilung erfolgt über kurze Wege direkt über die Rohdecke in den jeweiligen Raum der OP-Abteilung.

Die gewählte Trassenkonzeption gewährleistet die erforderliche Flexibilität und optimierte Raumhöhen in der OPAbteilung.

Weiterhin sind alle raumlufttechnischen Komponenten, wie z. B. OP-Umluftkühler, Brandschutzklappen, Volumenstromregler und Nacherhitzer gut zugänglich für Reinigungs-/Desinfektions-, Wartungsund Instandsetzungsarbeiten in der Technikzentrale untergebracht.

Durch die gewählte Andienung des OPBereichs direkt aus dem darüberliegenden Geschoss kann also der OP-Bereich selbst weitgehend frei von Schächten gehalten werden und dadurch ein Höchstmaß an Freiheit für die funktionell erforderliche Raumeinteilung gewährleistet werden. Sämtliche technischen Wartungsarbeiten werden in der OP-Zentrale vorgenommen ohne dass der OP-Bereich betreten werden muss. Es entstehen für die OP's geringere Stillstandszeiten und geringerer Aufwand bei der Desinfektion.

Jeder OP, der Sterilflur sowie die Nebenräume innerhalb der OP-Abteilung werden über separate Zonen angefahren, das heißt eine individuelle Konditionierung der einzelnen Bereiche ist gegeben.

Somit wird auch sichergestellt, dass zum Beispiel im Sterilflur später eine LaminarAir-Flow-Zuluftdecke installiert werden kann, um zukünftig die Vorbereitung des sterilen Instrumentariums durch eine großflächige LAF-Zuluftdecke weiter zu verbessern.

\section{Wirtschaftlichkeit}

Durch das gewählte System ergibt sich eine Minimierung der künftigen Betriebskosten unter anderem dadurch, dass im Gegensatz zu konventionellen/klassischen OP-Lüftungsdecken bei den in diesem Bauvorhaben vorgesehenen LaminarAir-Flow-Decken mit externen Umlufteinheiten nur jeweils maximal $1200 \mathrm{~m}^{3} / \mathrm{h}$ pro OP zentral aufbereitet und transportiert werden müssen. Durch das gewählte Konzept entfällt auch die Notwendigkeit des jährlichen Nachweises und der Dokumentation der Einhaltung eines permanenten gerichteten Druckgefälles über mehrere Raumbereiche.

Darüber hinaus lassen sich weiterhin Betriebskosteneinsparungen erzielen, weil außerhalb des OP-Betriebs die Umluftvolumenströme der OP-Decken zu 100\% abgeschaltet und die Außenluftvolumenströme ebenfalls um bis zu maximal $80 \%$ reduziert werden.

Es entfallen weiterhin die ansonsten regelmäßig erforderlichen Messungen für Narkosegase, da aufgrund der extrem hohen Luftwechsel im OP der zulässige MAK-Wert nicht überschritten wird. Eine weitere Besonderheit der LaminarAir-Flow-OP-Zuluftdecken in der BGU Frankfurt ist der umlaufende Umluftring, der im Gegensatz zu den oft verwendeten Umluftmodulen sicherstellt, dass die Umluft gleichmäßig im OP abgesaugt wird, anstatt örtlich konzentriert.

\section{Wartung}

Im Bereich der raumlufttechnischen Anlagen werden für die Durchführung von Wartungsarbeiten die Vorschriften „Krankenhaushygienische Leitlinien für die Ausführung und den Betrieb von raumlufttechnischen Anlagen“ (RLT-Anlagen) in Krankenhäusern, DIN 1946, T.4 (Raumlufttechnische Anlagen in Krankenhäusern) und die VDI 6022, T.1 (Hygienische Anforderungen an raumlufttechnische Anlagen - Komfortbereiche -) herangezogen.

Außerdem kann seit kurzem die neue VDIRichtlinie VDI 2167, T.1 (Entwurf), Technische Gebäudeausrüstung von Krankenhäusern - Heizungs- und Raumlufttechnik, verwendet werden, in der Wartungsrichtlinien nach einem Qualifizierungsverfahren abgeleitet werden können.

Der hygienische Betrieb von raumlufttechnischen Anlagen im OP ist wie bereits in den vorangestellten Abschnitten dargestellt bereits durch die planerische Grundkonzeption vorgegeben.

Im Bauprojekt BG Unfallklinik Frankfurt werden alle raumlufttechnischen Komponenten, wie z.B. OP-Umluftkühler, Brandschutzklappen, Volumenstromregler, Nacherhitzer, regelungstechnische Geräte gut zugänglich für Reinigungs-/ Desinfektions-, Wartungs- und Instandsetzungsarbeiten in der Technikzentrale untergebracht. Ein Öffnen der abgehängten Decken im OP-Bereich für Wartungszwecke ist nicht erforderlich.
Hierdurch werden die erforderlichen Reinigungs- und Desinfektionsmaßnahmen in o.g. Bereichen vereinfacht, sind in kürzerer Zeit und mit weniger Aufwand auszuführen. Die Stillstandszeit des OP‘s verringert sich hierdurch erheblich.

\section{Literatur}

1 DGKH, SGSH, OGHMP: Krankenhaushygienische Leitlinien für die Ausführung und den Betrieb von RLT-Anlagen in Krankenhäusern (2001), www.dgkh.de

2 DIN 1944 Teil 4 (1999-03): Raumlufttechnische Anlagen in Krankenhäusern

3 VDI 6022 Blatt 1, Ausgabe: 1998-07: Hygienische Anforderungen an Raumlufttechnische Anlagen- Büro- und Versammlungsräume, Komfortbereiche

4 VDI 2167 Blatt 1 (Entwurf, 2004-12): Technische Gebäudeausrüstung von Krankenhäusern - Heizungs- und Raumlufttechnik

${ }^{5}$ Lüderitz P, Lufthygienische Anforderungen im Krankenhaus und Abnahmeprüfungen von OP-Räumen nach VDI 2167/1 (SWK 99-3). VDI-Tagungsband 1870: TGA im Krankenhaus (2005). Vortrag Nr. 10.

6 Veröffentlichung „Modernisierung und $\mathrm{Er}$ weiterung des Städtischen Klinikum Brandenburg - OP-Abteilung - " Oktober 2003 Dipl.-Ing. (FH) Jürgen Trautwein, Rentschler und Riedesser Ingenieurgesellschaft $\mathrm{mbH}$ Zossener Straße 5510961 Berlin

\section{Dipl. Ing. Architekt Andreas Schmucker Generalplanung}

Schmucker + Partner

Planungsgesellschaft mbH, P 7, 14

D-68161 Mannheim

Dipl. Ing. (FH) Andreas Kuhnle

Projektleitung Haustechnik

Rentschler und Riedesser Ingenieurgesellschaft $\mathrm{mbH}$ für Technik im Bau

Filderbahnstrasse 12

D-70794 Filderstadt

\section{Dipl. Ing. (FH) Volker Williger}

Umbaukoordinator

WISAG Gebäude- und Betriebstechnik $\mathrm{GmbH} \&$ Co. KG

Rebstöcker Straße 35

D-60326 Frankfurt

Prof. Dr. Med. Reinhard Hoffmann Ärztlicher Direktor

B6-Unfallklinik

Friedberger Landstraße 430

D-60389 Frankfurt 